\title{
Some Characteristics of Vaginal Prolapse in Nepali Buffaloes
}

\author{
Shyam Kishore $\mathrm{SAH}^{1)}$ and Toshihiko $\mathrm{NAKAO}^{2) *}$ \\ ${ }^{1)}$ Department of Theriogenology, Institute of Agriculture and Animal Science, Rampur, Chitwan, Nepal and ${ }^{2)}$ Department of Development \\ Technology, Graduate School for International Development and Cooperation, Hiroshima University, Higashi-Hiroshima 739-8529, \\ Japan
}

(Received 19 April 2003/Accepted 29 July 2003)

ABSTRACT. Prolapse of vagina is one of the important maternal abnormalities during pregnancy in cattle and buffaloes. A field investigation was carried out on 26 Murrah graded buffaloes to study clinical characteristics of vaginal prolapse in buffaloes in Nepal. Fiftyseven percent of the 26 buffaloes with vaginal prolapse were either heifers or in first lactation. Sixty-five percent of the cases were in seventh month of pregnancy or later. About three quarters of the cases occurred between June and October. Twelve cases (63\%) of the 19 animals excluding 7 heifers had a history of vaginal prolapse in previous gestations. A half of the buffaloes were showing prolapse of the vagina even when they were in standing position and showing moderate or vigorous straining. After the conventional treat ments, twenty-three buffaloes retained the replaced vagina and calved normally. One animal aborted although the vagina was retained. Two buffaloes had severest degree of vaginal prolapse complicated with edema, injury and cyanosis, and they did not respond to the treatment. The two buffaloes had frequently recurrent prolapse and subsequently died. Early detection and prompt treatment may be imperative to control the vaginal prolapse in buffaloes.

KEY WORDS: buffaloe, pregnancy, vaginal prolapse.

The vagino-cervical prolapse constitutes a major problem of reproductive and subsequently of economic importance in cows and buffaloes [3,9] Mishandling of the prolapse results in irrepairable damage in the reproductive organs leading to infertility. The losses, however, can be minimized to a great extent through timely and appropriate interventions [9]. The incidence varies according to the stage of pregnancy, breed and season [8]. No information, however, is available on the incidence and characteristics of this condition in buffaloes in Nepal. Objective of the present study was therefore to show some characteristics of vaginal prolapse in Nepalese buffaloes as related to parity, stage of pregnancy and degree of prolapse, complications, treatment, and prognosis.

\section{MATERIALS AND METHODS}

This study was conducted between April 2000 and December 2001 on different farms in the Chitwan District of Nepal. The chance to collect cases was half in the month of January, February and March in comparison with other months of the year. A total of 26 Murrah or graded buffaloes were diagnosed as having vaginal prolapse. Information about parity of the animals, history of vaginal prolapse, date of the last mating and date of the occurrence of the vaginal prolapse was obtained from the farmers.

Cases of vaginal prolapse were classified into four groups according to the severity of the conditions [9].

Degree I: Only a part of vaginal prolapse appears in a lying buffalo.

\footnotetext{
* Correspondence to: NaKao, T., Graduate School for International Development and Cooperation, Hiroshima University, Kagamiyama 1-5-1, Higashi-Hiroshima 739-8529, Japan.
}

Degree II: Whole vagina was prolapsing through vulvar lips in a lying buffalo .

Degree III: The whole vagina (Cervical os visible) was prolapsing through vulvar lips even when the animals were standing.

Degree IV: The whole vagina (Cervical os visible) was prolapsing through vulvar lips in standing animals and had inflammation, injuries, edema and cyanosis. Animal were straining continuously.

Treatments: The vaginal prolapse in each buffalo was washed with $5 \%$ acriflavin once daily for 3 days. In addition, the buffaloes with Degree I Vaginal prolapse were made to stand/ sit on a floor having 20-30 cm slope towards anterior. Buffaloes with vaginal prolapse of Degree II to IV were given epidural anaesthesia with 7 to $10 \mathrm{ml} 2 \%$ xylocain and the prolapse was reposed after being dressed with antiseptic creams, vegetable oil and gruel. A rope truss was applied to try to keep the prolapsed mass in position after replacement; then, calcium $(20 \%)$ solution, $1,000 \mathrm{ml}$, was injected intravenously to cause the contraction of the uterus. The buffaloes under Degree IV was also injected once with xylazine $(1.5 \mathrm{ml})$ intra- muscularly to relax straining in order to prevent recurrence of prolapse. In case xylazine was not effective, deposit type of progesteron $500 \mathrm{mg}$ was injected twice weekly. The treatments like antiseptic wash, application of ointments, truss and even xylocain epidural were repeated daily until complete cessation of the prolapse.

\section{RESULTS}

Parity and stage of gestation in buffaloes with vaginal prolapse: Fifty-seven percent of buffaloes showing vaginal prolapse were either pregnant heifers or in the first lactation. 
Table 1. Relationship between days after last mating and degree of vaginal prolapse in buffaloes

\begin{tabular}{ccccccccc}
\hline Degree* & $90-120$ & $121-150$ & $151-180$ & $181-210$ & $211-240$ & $241-270$ & $271-300$ & $301-307$ \\
\hline I & 1 & 0 & 2 & 0 & 2 & 0 & 1 & 1 \\
II & 0 & 1 & 0 & 0 & 0 & 0 & 3 & 0 \\
III & 0 & 0 & 1 & 2 & 2 & 2 & 4 & 0 \\
IV & 1 & 0 & 0 & 0 & 0 & 1 & 0 & 0 \\
Total & 2 & 1 & 3 & 2 & 4 & 3 & 8 & 1 \\
\hline
\end{tabular}

* See Table 2 for description of degree of vaginal prolapse.

Note: Out of 26 buffaloes with vaginal prolapse, 2 were not pregnant and, therefore, excluded from the table.

Distribution of the cases by stage of gestation is presented in Table 1. Of 26 buffaloes with vaginal prolapse two were not pregnant and, therefore, not included in the table. The buffaloes at 7 month of pregnancy or later accounted for $66.7 \%$ of the total number of cases.

Seasonal occurrence of vaginal prolapse in buffaloes: Seventy-seven percent of the cases were presented between June and October with highest proportion occurring in August.

Previous history of vaginal prolapse: Twelve buffaloes (63\%) out of the 19 buffalo cows had the history of having vaginal prolapse in the previous gestations.

Degree of vaginal prolapse: Twelve $(46.2 \%)$ of the 26 buffaloes were classified as vaginal prolapse of Degrees I and II. The other $14(53.8 \%)$ were classified as vaginal prolapse of Degree III and IV (Table 2). Table 1 shows the relationship between days after last mating and degree of vaginal prolapse. No relationship was seen between the two parameters.

Edematous swelling of the prolapsed vagina of different severity was observed in $10(52.6 \%)$ of the 19 cases with vaginal prolapse of Degree II to IV. While no vaginal edema was seen in 7 animals with vaginal prolapse of Degree I (Table 3). Of the 5 cases with Degree II prolapse, three showed slight edema and two had moderate swelling. Fourty one point seven percent of the Degree III cases showed moderate or severe edematous swelling of the prolapsed part. Both of the two buffaloes with Degree IV vaginal prolapse had severe edematous swelling and had injury to the prolapsed vagina and showed cyanotic changes.

Almost all buffaloes with vaginal prolapse of Degree III and IV showed moderate to severe continuous straining (Table 3). The degree of straining increased with the degree
Table 2. Degree of vaginal prolapse in buffaloes

\begin{tabular}{lcc}
\hline Degree of prolapse & No. of cases & Percentage \\
\hline I: A part of vagina when lying & 7 & 26.9 \\
II: Whole vagina when lying & 5 & 19.2 \\
III: Whole vagina even when standing & 12 & 46.2 \\
IV: Whole vagina with complications & 2 & 7.7 \\
\hline
\end{tabular}

of severity. None of the cases with Degree I showed straining. All the buffaloes were having normal appetite except the one with severe straining.

Prognosis of buffaloes with vaginal prolapse: Of the 26 buffaloes with different degrees of vaginal prolapse, 23 $(88.5 \%)$ retained the replaced vagina after three consecutive days of treatments and subsequently calved normally (Table 4). One buffalo aborted three weeks after the last treatment while the replaced vagina was retained. Vaginal prolapse recurred in two animals 10 to 11 days later after replacing the prolapsed vagina following treatments for two consecutive days and these buffaloes died five days after reccurrence of vaginal prolapse. They were with severe straining, arched back, eyes red, deep shallow respiration, off feed and water, gradually recumbent, ultimately collapsed. In one of the cases (No. 10), the hind quarter was cleaned with $5 \%$ acriflavin solution, xylocain $2 \%-10 \mathrm{~m} l$ was injected epidurally, the prolapsed mass was reposed in position and a truss was applied. Calcium $(20 \%, 1,000 \mathrm{ml})$ solution was injected intravenously. The hind quarter was raised for 3 days. The second case (No. 16) severely affected was treated as above, in addition this buffalo was injected with $1.5 \mathrm{~m} l$ of xylazine to reduce straining. These two cases (Nos. 10 and 11), after the effect of xylocain and xylazine was over, again showed the straining gradually increasing to

Table 3. Edematous swelling of the vagina and straining in buffaloes with vaginal prolapse

\begin{tabular}{|c|c|c|c|c|c|c|c|}
\hline \multirow{2}{*}{ Degree* } & \multirow{2}{*}{$\begin{array}{c}\text { No. of } \\
\text { Animals }\end{array}$} & & \multicolumn{2}{|c|}{ Edematous swelling } & \multicolumn{3}{|c|}{ Straining } \\
\hline & & & Number & $\begin{array}{c}\text { Percentage } \\
(\%)\end{array}$ & & Number & $\begin{array}{c}\text { Percentage } \\
(\%)\end{array}$ \\
\hline I & 7 & & 0 & 0 & & 0 & 0 \\
\hline II & 5 & Slight & 3 & 60 & Weak & 2 & 40 \\
\hline \multirow[t]{2}{*}{ III } & 12 & Moderate & e 4 & 33.3 & Moderate & e 10 & 83.3 \\
\hline & & Severe & 1 & 8.3 & Strong & 1 & 8.3 \\
\hline IV & 2 & Severe & 2 & 100 & Strong & 2 & 100 \\
\hline Total & 26 & & 10 & 38.5 & & 15 & 57.7 \\
\hline
\end{tabular}

* See Table 2 for description of degree of vaginal prolapse. 
Table 4. Results of treatment for vaginal prolapse in buffaloes

\begin{tabular}{lccccc}
\hline & \multicolumn{5}{c}{ Degree of vaginal prolapse* } \\
& I & II & III & IV & Total (\%) \\
\hline No. of cows & 7 & 5 & 12 & 2 & $26(100)$ \\
Restored and settled & 7 & 5 & 11 & 0 & $23(88.5)$ \\
Restored but aborted & 0 & 0 & 1 & 0 & $1(3.8)$ \\
Recurred and died & 0 & 0 & 0 & 2 & $2(7.7)$ \\
\hline
\end{tabular}

* See Table 2 for description of degree of vaginal prolapse.

severity the following day. Postpartum pyometra was diagnosed in 1 buffalo which was corrected with treatments later.

\section{DISCUSSION}

Majority of vaginal prolapse in cattle is seen in the last two months of gestation [1]. A similar tendency was also observed in buffaloes in this study. Sixty-five percent of the cases were in seventh month of pregnancy or later. The exact cause of vaginal prolapse is not known. However the increased intra-abdominal pressure due to the fetal growth and placental development and softening of the genital ligaments due to the increased estrogen synthesis in the placenta in the later part of gestation may cause vaginal prolapse. A substantial number of the cases of vaginal prolapse were observed in the second trimester of gestation period in this study, which suggests that there might have been some other causes of vaginal prolapse.

Buffaloes with prepartum prolapse of vagina had significantly lower concentrations of calcium and phosphorous in blood serum compared to their normal counterparts. The serum progesterone concentrations of these buffaloes at various stages of pregnancy, however, was similar to the normal [4]. Sixty-three percent buffaloes in this study had a history of vaginal prolapse in preceeding gestations which suggests its genetic origin. In India, repetition of the prepartum prolapse of vagina in successive pregnancies was observed in about $50 \%$ buffaloes which suggested that the condition could be of genetic origin [3]. Vaginal proplapse in buffaloes has a heritability of $0.33 \pm 0.13$, and repeatability of 0.42 [11].

All buffaloes with vaginal prolapse were given vaginal douche with $1 \%$ potassium permanganate solution followed by application of furacin antiseptic cream. In addition, the buffaloes were treated with intravenous infusion of $20 \%$ calcium borogluconate solution or were given progesterone injections every week for three weeks. The calcium therapy provided only temporary relief from the prolapse. The exogenous progesterone treatment reduced tenesmus and improved overall management of clinical cases probably by counteracting the excessive relaxation of pelvic ligaments and peri-vaginal tissue [4]. Fifty-seven percent of the cases of vaginal prolapse in buffaloes were heifers or primiparous cows in this study. The same trend in buffaloes was reported in India [10]. As the heifers and primiparous having less extended/voluminous abdominal space and pelvic spaces than that of pluriparous buffaloes during pregnancy may cause more prone to suffer from prolapse of vagina.

Seasonality in the occurrence of vaginal prolapse in buffaloes was seen in the present investigation. This may be due to the seasonal breeding pattern of buffaloes $[2,10]$, as most of the calvings are seen during this period. The highest incidence of prolapse occur during rainy season which may be the result of the indirect effect of climatic and environmental conditions. Still further investigations are necessary for the etiological factors of prepartum vaginal prolapse in the buffaloes.

Treatment of vaginal prolapse using generally recommended techniques was effective in 23 of the 26 buffaloes in this study. Recurrence of the prolapse can also be controlled in cows by the surgical occlusion of the Caslick's operation or the subcutaneous suture of nylon tape around the vulva known as Buhner's method [1, 5-7]. However, the surgical treatment was not conducted here because the instruments and materials for the suture was not locally available and the farmers could not have cared properly about hygienic conditions of the sutured vulva.

ACKNOWLEDGEMENT. This study was partly supported by Grant-in-Aid for Scientific Research (B)(2) from the Ministry of Education, Culture, Sports, Science and Technology, Japan.

\section{REFERENCES}

1. Arthur, G.H. 1996. Prolapse of the vagina. pp. 134-140. In: Veterinary Reproduction and Obstetrics, 7th ed. (Arthur, G.H., Noakes, D.E., Pearson, H. and Parkinson, T.J. eds.), W.B. Saunders Co., Ltd., London.

2. Mishra, U.K. and Agrawal, R.G. 1998. Incidence of prolapse of genitals in Murrah buffalo in relation to season, pregnancy, parity and management. Ind. Vet. J. 75: 254-255.

3. Nanda, A.S. and Sharma, R.D. 1982. Incidence and etiology of prepartum prolapse of vagina in buffalo. Ind. J. Dairy Sci. 35: 168-171.

4. Nanda, A.S. and Sharma, R.D. 1981. Study on the calcium and progesterone treatment in prepartum prolapse of vagina in buffalo. Ind. J. Anim. Res. 15: 37-40.

5. Norton, C.S. 1969. External fixation of bovine vagina after reduction of prolapse. J. Am. Vet. Med. Assoc. 154: 11791181.

6. Pierson, R.E. 1961. A review of surgical procedure for correction of vaginal prolapse in cattle. J. Am. Vet. Med. Assoc. 139: 352-356.

7. Rai, C.S. and Prabhakar, S. 2000. Clinical effects of epidural administration of xylazine in buffaloes having prepartum vaginal prolapse. Ind. Vet. J. 77: 247-249.

8. Reddy, V.N. Viswanatha, 1994. Reproductive Disorders in Dairy Cows. V.S. Publication, Bangalore, India.

9. Roberts, S.J. 1986. Veterinary Obstetrics and Genital Disease. 3rd ed., David and Charles Inc., Vermont.

10. Sheth, R.A. 1966. Some observations about incidence of prolapse of vagina in Surti buffaloes. Ind. Vet. J. 47: 1130-1134.

11. Tomar, S.S. and Tripathi, V.N. 1992. Estimates of heritability of utero-vaginal disorders in a herd of Murrah buffalo. Ind. J. Anim. Sci. 62: 663-664. 